\title{
Semi-Blind and Group-Blind Multiuser Detection for the MC-CDMA Uplink
}

\author{
Hua Wei and Lajos Hanzo ${ }^{1}$ \\ School of ECS, University of Southampton, SO17 1BJ, UK. \\ Tel: +44-23-8059-3125, Fax: +44-23-8059-4508 \\ Email:1h'@ecs.soton.ac.uk, http://www-mobile.ecs.soton.ac.uk
}

\begin{abstract}
In this contribution both semi-blind and groupblind multiuser detectors designed for uplink MCCDMA systems are investigated in conjunction with different spreading codes. In the investigated scenario Walsh codes are shown to outperform both Gold codes and Zadoff-Chu codes. A short cyclic prefix, rather than differential coding, is : used for removing the phase ambiguity imposed by the blind channel estimation scheme, and the Akaike information theoretic criterion advocated by Wax and Kailath is invoked for the estimation of the rank of the semi-blind detectors, which is defined as the number of users activated in the channel. When supporting a total of $K=10$ users and exploiting the knowledge of the signature waveforms for the group of $\tilde{K}=7$ users communicating within the reference cell considered, while dispensing with any knowledge about the parameters of the remaining $K-\tilde{K}=3$ users roaming in the surrounding cells, the system's performance was found to be close to that achieved with the aid of perfect knowledge of all the signature waveforms, the channel coefficients and the total number of users $K$ supported. The achievable performance of this scenario was about $3 \mathrm{~dB}$ from the single user performance at a BER of $10^{-3}$.
\end{abstract}

\section{INTRODUCTION}

The philosophy of Multicarrier Code Division Multiple Access (MC-CDMA) systems [1] is based on a novel combination of CDMA and Orthogonal Frequency Division Multiplexing (OFDM) [2]. MC-CDMA constitutes a promising candidate for employment in the next generation

Acknowledgements: The work reported in this paper has formed part of the Wireless Enabling Techniques work area of the Core 2 Research Programme of the Virtual Centre of Excellence in Mobile and Personal Communications, Mobile VCE, www.mobilevce.com, whose funding support, including that of EPSRC, is gratefully acknowledged. Fully detailed technical reports on this research are available to Industrial Members of Mobile VCE. of wireless systems owing to its high grade of flexility, since it has a higher number of reconfigurable parameters, than classic single-carrier CDMA [1]. However, the capacity of MC-CDMA is limited by the effects of the multiuser interference (MUI). Hence, a range of Multiuser Detection (MUD) techniques [3-5] has been proposed in the literature for reducing its effects. For example, the Minimum Mean Square Error (MMSE) MUD has been characterized in $[1,3]$, while an Interference Cancellation (IC) based MUD has been proposed in $[1,4]$.

Subspace based blind multiuser detectors were first proposed by Wang and Poor [6] for single-carrier DSCDMA systems communicating over synchronous AWGN channels. Wang and Poor [7] later extended their work to dispersive asynchronous CDMA environments, where also a subspace based channel estimation approach was proposed. In $[8,9]$, Wang and Host-Madsen proposed group-blind multiuser detectors for uplink single-carrier DS-CDMA, which exploited the prior knowledge of all signature waveforms, rather than only that of the reference user. Their group-blind multiuser detector exhibited a substantial performance improvement over that of a blind multiuser detector.

In this contribution, we investigated the application of subspace-based semi-blind and group-blind multiuser detectors designed for the MC-CDMA uplink, while a short cyclic prefix, rather than differential encoding was used for removing the phase ambiguity encountered, because differential encoding sacrifices $3 \mathrm{~dB}$ in terms of the required Signal-to-Noise Ratio (SNR). The SNR loss owing to the transmission of the cyclic prefix is significantly lower. Furthermore, the Akaike information theoretic criterion (AIC) [10] was invoked for the estimation of the rank of the signal space, i.e. for estimating the number of users supported. As alternative design options, both a blind MMSE multiuser detector [6] and a form-II groupblind multiuser detector [8] were studied in conjunction with Zadoff-Chu spreading codes [11], Gold codes [12] and Walsh codes [12].

This paper is organized as follows. Section 2 introduces the MC-CDMA system model using a short cyclic prefix, while Section 3 characterizes the semi-blind and 
group-blind multiuser detectors studied. In Section 4 our simulation results are presented, while in Section 5 we offer our conclusions.

\section{SYSTEM MODEL}

Let us consider the employment of a $K$-user MC-CDMA system in the uplink, where $\tilde{K}$ of the users are in the reference cell. We assume furthermore that the signature waveforms of this group of $\tilde{K}$ users are known, while those of the $\bar{K}=K-\tilde{K}$ other-cell users are unknown. BPSK modulation was invoked. The modulating symbols $b_{k} \in\{-1,+1\}$ having a duration of $T_{b}$ are first spread using a spreading sequence $\mathbf{c}_{k}=\left[c_{k, 1}, c_{k, 2}, \ldots, c_{k, N}\right]$ of length $N$, where we have $\mathbf{c}_{k} \cdot \mathbf{c}_{k}^{H}=1$. For simplicity, in our investigations we assume that OFDM using $N$ subcarriers was invoked [2], where $N$ consecutive chips of the MC-CDMA spreading sequences were mapped to $N$ different subcarriers during an OFDM symbol and hence the OFDM symbol duration was $T_{s}=T_{b}$. The spread OFDM bit-vector of $N$ subcarriers can be expressed as $\mathbf{s}_{k}=\mathbf{c}_{k} b_{k}=\left[s_{1}, s_{2}, \ldots s_{N}\right]$. Then the Inverse Fast Fourier Transform (IFFT) is invoked for modulating the $N$ subcarriers by the spread information bits $\mathbf{s}_{k}$ [2]. This signal is transmitted through a multipath fading channel, which is assumed to have $L$ paths, hence the Channel Impulse Response (CIR) can be expressed as:

$$
h_{k}(t)=\sum_{\ell=0}^{L-1} h_{k, \ell} \cdot \delta\left(t-m T_{c}\right),
$$

where $h_{k, \ell}$ is the complex channel gain experienced by the signal of the $k$ th user in the $\ell$ th path, which obeys Rayleigh fading, while $T_{c}=T_{b} / N$ is the chip-duration. We assume that a sufficiently long cyclic prefix is incorporated in the OFDM symbol prior to transmission for the sake of compensating for both the asynchronous delay differences of the different users as well as for the delayspread-induced inter-OFDM-symbol interference imposed by the dispersive channel [2]. At the receiver, the samples of the received signal corresponding to the cyclic prefix are first removed and $N$-point FFT is invoked for demodulating the remaining $N$ samples [2]. Hence the received signal can be expressed in vectorial form as $\mathbf{r}=$ $\left[r_{1}, r_{2}, \ldots, r_{N}\right]$

$$
\mathbf{r}=\sum_{k=1}^{K} \mathbf{S}_{k} \cdot \mathbf{H}_{k}+\mathbf{n}=\mathbf{G} \cdot \mathbf{b}+\mathbf{n},
$$

where $\mathbf{H}_{k}=\left[H_{k, 0}, H_{k, 1}, \ldots, H_{k, N}\right]$ denotes the frequency domain channel transfer function, and $\mathbf{S}_{k}$ is a diagonal matrix given by $\mathbf{S}_{k}=\operatorname{diag}\left\{\mathbf{s}_{k}\right\}$. Since we assume that a sufficiently long cyclic prefix was inserted, no OFDM intersymbol interference (ISI) is incurred. Hence $\mathbf{H}_{k}$ can be expressed as the $N$-point DFT of $\mathbf{h}_{k}=\left[h_{k, 0}, \ldots, h_{L-1}\right]^{T}$, where the operator [ $]^{T}$ denotes the matrix transpose. More explicitly, we have $\mathbf{H}_{k}=\mathbf{F}_{L} \cdot \mathbf{h}_{k}$, where $\mathbf{F}_{L}$ is an $(N \times L)$-dimensional matrix, which is given by the first $L$ columns of the DFT matrix $\mathbf{F}$ formulated as:

$$
\mathbf{F}=\left(\begin{array}{cccc}
1 & 1 & \ldots & 1 \\
1 & e^{-j 2 \pi / N} & \ldots & e^{-j 2 \pi(N-1) / N} \\
\vdots & \vdots & \ddots & \vdots \\
1 & e^{-j 2 \pi(N-1) / N} & \cdots & e^{-j 2 \pi(N-1)(N-1) / N}
\end{array}\right)
$$

Hence the effective signature waveform of the $k$ th user, which is constituted by the convolution of the spreading codes and the CIR, can be expressed as: $\mathbf{g}_{k}=\mathbf{C}_{k} \mathbf{H}_{k}$, where $\mathbf{C}_{k}=\operatorname{diag}\left\{\mathbf{c}_{k}\right\}$. The matrix $\mathbf{G}$ in Eq.(2) is an $(N \times K)$-dimensional matrix comprising the convolution of the channel's complex fading factors and the spreading signatures of all the $K$ users, hence for the combined CIR we have $\mathbf{G}=\left[\mathbf{g}_{1}, \mathbf{g}_{2}, \ldots, \mathbf{g}_{K}\right]$. The vector $\mathbf{b}=\left[b_{1}, b_{2}, \ldots, b_{N}\right]^{T}$ is the data vector and $\mathbf{n}$ is the white Gaussian noise vector associated with the covariance matrix of $\sigma^{2} \mathbf{I}_{N}$, where $\mathbf{I}_{N}$ denotes the $(N \times N)$-dimensional identity matrix.

\section{THE SEMI-BLIND RECEIVER}

In this section we will consider subspace based blind channel estimation, as well as semi-blind and group-blind linear multiuser detection invoking rank estimation. Let us first consider the underlying blind channel estimation philosophy.

\subsection{Blind Channel Estimation}

When the background noise is white, we may invoke eigenanalysis of the autocorrelation matrix of the received MC-CDMA signal vector $\mathbf{r}$. Upon extending the singlecarrier DS-CDMA philosophy of $[6,7]$ to our MC-CDMA scenario, the corresponding eigendecomposition is given by:

$$
\begin{aligned}
\mathbf{R} & =E\left\{\mathbf{r r}^{H}\right\}=\mathbf{G G}^{H}+\sigma^{2} \mathbf{I}_{N} \\
& =\mathbf{U}_{s} \boldsymbol{\Lambda}_{s} \mathbf{U}_{s}^{H}+\sigma^{2} \mathbf{U}_{n} \mathbf{U}_{n}^{H},
\end{aligned}
$$

where the superscript ( $)^{H}$ denotes the conjugate transpose of a matrix. Since the matrix $\mathbf{G}$ has a full column rank of $r=K$, the matrix $\mathbf{G G}^{H}$ in Eq.(4) has a rank of $r$. Therefore, in Eq.(4) $\boldsymbol{\Lambda}_{s}=\operatorname{diag}\left(\lambda_{1}, \lambda_{2}, \ldots, \lambda_{r}\right)$ contains the $r$ largest eigenvalues of $\mathbf{R}$ in descending order (i.e. we have $\left.\lambda_{1} \geq \lambda_{2} \geq \ldots \geq \lambda_{r}>\sigma^{2}\right), \mathbf{U}_{s}=\left[\mathbf{u}_{1} \ldots \mathbf{u}_{r}\right]$ contains the corresponding orthonormal eigenvectors, while $\mathbf{U}_{n}=\left[\mathbf{u}_{r+1} \ldots \mathbf{u}_{N}\right]$ contains the $(N-r)$ number of orthonormal eigenvectors that correspond to the eigenvalue $\sigma^{2}$. The channel response $\mathbf{h}_{k}$ can be estimated by exploiting the orthogonality between the signal space and 
the noise space. Specifically, since $\mathbf{U}_{n}$ is orthogonal to the column space of $\mathbf{G}$, while $\mathbf{g}_{k}$ is in the column space of $\mathbf{G}$, we have:

$$
\mathbf{U}_{n}^{H} \mathbf{g}_{k}=\mathbf{U}_{n}^{H} \mathbf{C}_{k} \mathbf{F}_{L} \mathbf{h}_{k}=0 .
$$

Hence, an estimate of the channel response $\mathbf{h}_{k}$ can be obtained by computing the minimum eigenvector of the ma$\operatorname{trix}\left(\mathbf{F}_{L}^{H} \mathbf{C}_{k}^{H} \mathbf{U}_{n} \mathbf{U}_{n}^{H} \mathbf{C}_{k} \mathbf{F}_{L}\right)$, which constitutes a necessary condition for such a channel estimate to be unique, when we have $L \leq N-K$. Furthermore, note that there is an arbitrary phase ambiguity in the estimate of the channel response, but we can remove this specific phase ambiguity with the aid of the cyclic prefix using the technique proposed in [13].

\subsection{Rank Estimation}

Based on the estimated eigenvalues $\Lambda=\left[\lambda_{1}, \lambda_{2}, \ldots\right.$, $\lambda_{N}$ ] and using the Akaike information theoretic criterion (AIC) [10], the rank of the signal space (i.e. the number of active users in the system ), can be estimated with the aid of [10]. The quantities invoked in the AIC are defined as follows:

$$
\operatorname{AIC}(k)=(N-k) \cdot M \cdot \ln \alpha(k)+k \cdot(2 N-k),
$$

where $M$ is the number of data bits used for the estimation of the total number of users supported, namely $K$, where $M$ is conveniently chosen to be the transmission burst length, and $\alpha(k)$ can be expressed as [10]:

$$
\alpha(k)=\frac{\left(\sum_{i=k+1}^{N} \lambda_{i}\right) /(N-k)}{\left(\Pi_{i=k+1}^{N} \lambda_{i}\right)^{1 /(N-k)}} .
$$

The estimate $\hat{K}$ of the rank $K$ of the signal space is given by that particular value of $k$, which minimizes Eq.(6). Hence we have

$$
\hat{K}=\arg \min _{0 \leq k \leq N-1}\{\operatorname{AIC}(k)\} .
$$

\subsection{Semi-Blind and Group-Blind Multiuser De- tection}

A linear detector invoked for user $k$ can be represented by the MUD's weight vector $\mathbf{w}_{k} \in C^{N}$, which is applied to the received signal $\mathbf{r}$, such that the decision related to $b_{k}$ may be expressed as:

$$
\hat{b}_{k}=\operatorname{sgn}\left\{\mathbf{w}_{k}^{H} \mathbf{r}\right\} \text {. }
$$

The linear semi-blind MMSE multiuser detector, which assumes only the knowledge of the signature waveform of the reference user but not of the other users is given by [6]:

$$
\mathbf{w}_{k}=\frac{1}{\mathbf{g}_{k}^{T} \mathbf{U}_{s} \boldsymbol{\Lambda}_{s}^{-1} \mathbf{U}_{s}^{H} \mathbf{g}_{k}} \mathbf{U}_{s} \boldsymbol{\Lambda}_{s}^{-1} \mathbf{U}_{s}^{H} \mathbf{g}_{k} .
$$

In contrast to this semi-blind multiuser detection philosophy, the group-blind multiuser detector exploits the knowledge of a group of $\tilde{K}$ composite signature waveforms. More specifically, let us define the set $\overline{\mathbf{G}}$ such that $\tilde{\mathbf{G}}=\left[\mathbf{g}_{1} \ldots \mathbf{g}_{\tilde{K}}\right]$ is an $(N \times \tilde{K})$-dimensional matrix, which includes the signature waveforms of the group of $\tilde{K}$ users communicating within the cell of the reference user. The group-blind linear hybrid detector of user $k, k=1, \ldots, \tilde{K}$, is given by the solution of the following constrained optimization problem [8]:

$$
\begin{aligned}
& \mathbf{w}_{k}=\quad \arg \min _{\mathbf{w}_{k} \in \operatorname{range}(\mathbf{G})} E\left\{\left\|b_{k}-\mathbf{w}_{k} \mathbf{r}\right\|^{2}\right\} \\
& \text { subject to } \mathbf{w}_{k} \tilde{\mathbf{G}}=\mathbf{1}_{k}^{T}, \quad k=1, \ldots, \tilde{K},
\end{aligned}
$$

where $1_{k}$ is the vector having a length of $\tilde{K}$ and having elements of zero, except for the kth element, which is 1 . Generally speaking, this detector invokes zero-forcing of the interference caused by the group of $\tilde{K}$ users whose signature sequences are known, and suppresses the interference imposed by the other-cell users, whose signature sequences are unknown, employing the MMSE criterion. The so-called form-II solution [8] and the corresponding bit estimate of user $k$ may be written as [8]:

$$
\begin{aligned}
\mathbf{w}_{k} & =\mathbf{U}_{s} \boldsymbol{\Lambda}_{s}^{-1} \mathbf{U}_{s}^{H} \tilde{\mathbf{G}}\left[\tilde{\mathbf{G}}^{H} \mathbf{U}_{s} \boldsymbol{\Lambda}_{s}^{-1} \mathbf{U}_{s}^{H} \tilde{\mathbf{G}}\right]^{-1} \mathbf{1}_{k} \\
\hat{b}_{k} & =\operatorname{sgn}\left\{\operatorname{Re}\left(\mathbf{w}_{k}^{H} \mathbf{r}\right)\right\}, \quad k=1, \ldots, \tilde{K} .
\end{aligned}
$$

Hence, we require the knowledge of the composite signature waveforms of the group of $\tilde{K}$ users supported within the reference cell, in order to construct $\tilde{\mathbf{G}}$. Finally, the operation of this receiver is summarized in Table 1.

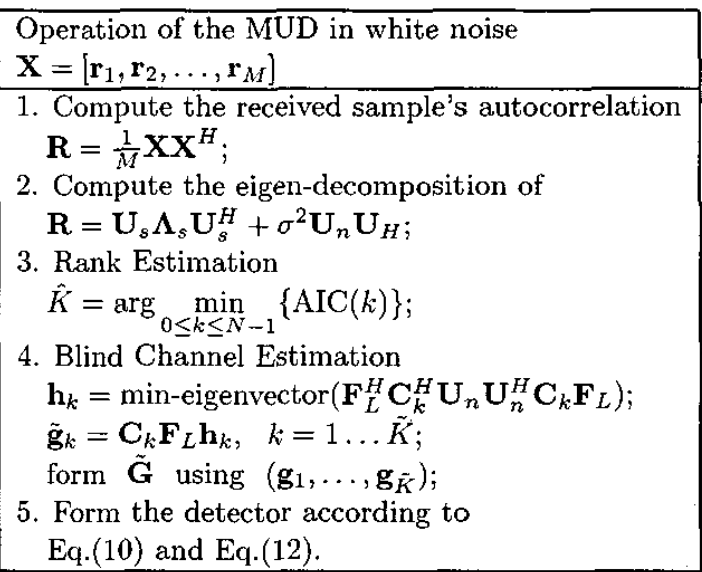

Table 1: The operation of the blind and group-blind MCCDMA multiuser detector communicating over a dispersive AWGN channel. 


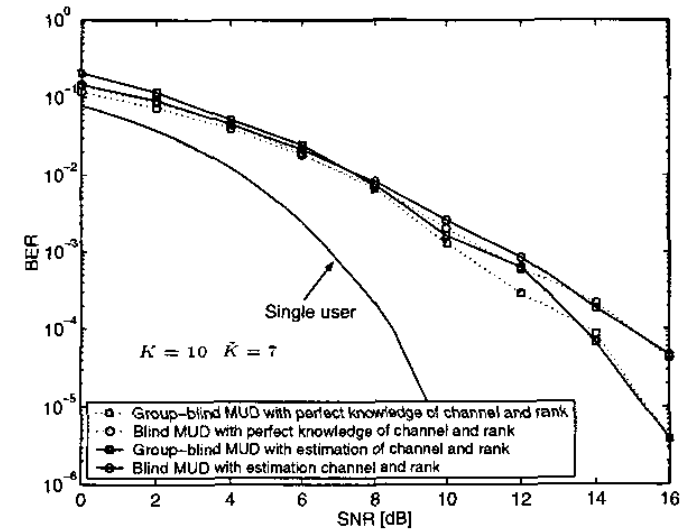

Figure 1: BER performance of the various detectors considered. Both the scenario of having a perfect knowledge of the complex-valued channel coefficient and the rank as well as their imperfect estimation was characterized. Walsh codes having a spreading factor of $N=16$ were employed and $K=10, \tilde{K}=7$ users were supported. All system parameters are summarized in Table 2.

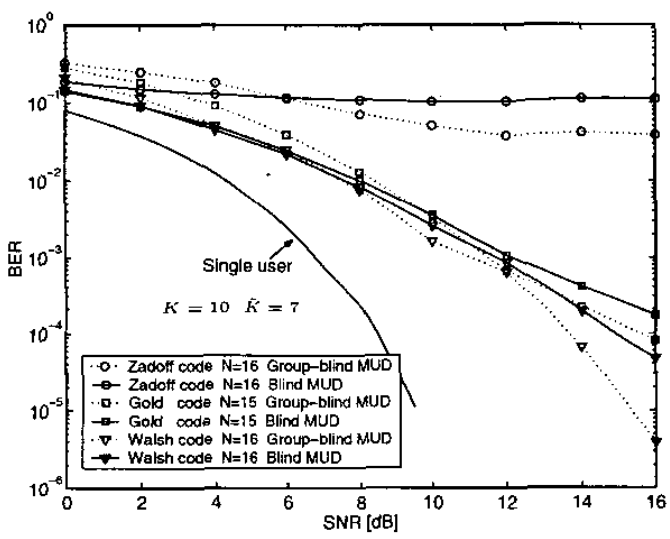

Figure 2: BER performance comparison of blind and group-blind multiuser detectors, when Walsh, Gold and Zadoff-Chu codes were invoked and $K=10, \tilde{K}=7$ users were supported. All system parameters are summarized in Table 2 .

\begin{tabular}{|l|l|}
\hline Parameters & Value \\
\hline CIR length $L$ & 3 \\
\hline Normalized Dopper frequency & 0.01 \\
\hline Short cyclic prefix & 2 chips \\
\hline Burst length $M$ & 256 \\
\hline
\end{tabular}

Table 2: The basic simulation parameters for the semiblind and group-blind multiuser detectors

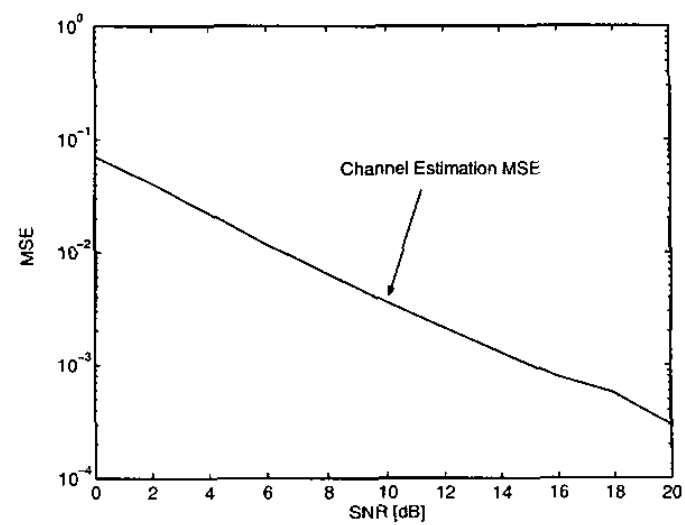

Figure 3: MSE performance of the blind channel estimator, when Walsh codes having a spreading factor of $N=16$ were employed and $K=10$ users were supported. All system parameters are summarized in Table 2.

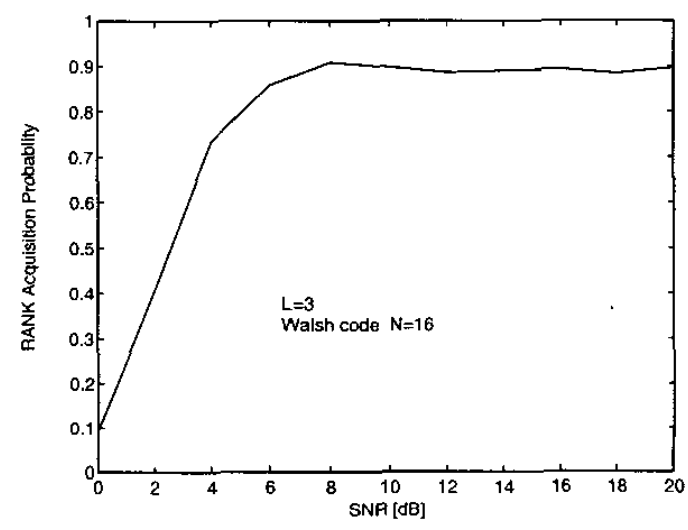

Figure 4: Correct rank acquisition probability, when Walsh codes having a spreading factor of $N=16$ were employed and $K=10$ users were supported. All system parameters are summarized in Table 2.

\section{SIMULATION RESULTS}

In this section we provide computer simulation results for characterizing the performance of the proposed blind and group-blind multiuser detectors, when communicating over a multipath Rayleigh fading channel having $L=$ 3 paths. More specifically, the fading factors of each complex-valued path are generated according to the Rayleigh distribution, where the paths are normalized so that each user's signal arrives at the receiver with an equal power, i.e. we have $\left\|\mathbf{h}_{k}\right\|^{2}=1$. The complex-valued fading coefficients are assumed to be static during a block of $M=256 \mathrm{MC}$-CDMA bits, which is the transmission burst length. 
In Fig. 1 we observe that both the semi-blind and group-blind multiuser detectors are capable of achieving a performance, which is close to that associated with a perfect knowledge of the channel coefficients and the number of users $K$. More specifically, the BER performance degradation incurred by the imperfect channel estimation technique of Section 3.1 and rank estimation algorithm of Section 3.2 was found negligible.

Fig. 2 characterizes the BER performance of the semiblind and group-blind multiuser detectors considered in conjunction with different spreading codes. Observe that the performance of the Zadoff-Chu codes

[2] is the worst, while Walsh codes achieved the best performance.

Finally, Fig. 3 portrays the Mean Square Error (MSE) performance of the subspace-based channel estimator of Section 3.1, while Fig. 4 characterizes the achievable correct rank acquisition probability based on the AIC algorithm of Section 3.2 .

\section{CONCLUSION}

In this contribution, we investigated the performance of semi-blind and group-blind multiuser detector schemes in the context of the MC-CDMA uplink using a short cyclic prefix, which assisted us in removing the ISI and the phase ambiguity associated with the blind channel estimation process. In our study, both the blind and group-blind multiuser detectors were shown to be capable of achieving a similar performance to that attained with the aid of perfect knowledge of the channel coefficients, spreading codes and the rank information, i.e. the number of users $K$ supported. Furthermore, the Walsh spreading code based MC-CDMA scheme outperformed the corresponding Gold and Zadoff-Chu code based systems. Our future work will consider the employment of blind and group-blind multiuser detectors in the context of the MCCDMA uplink dispensing with the use of a cyclic prefix as well as in conjunction with sophisticated channel codes.

\section{REFERENCES}

[1] L. Hanzo, L. L. Yang, E. L. Kuan, and K. Yen, Single- and Multi-Carrier DS-CDMA. John Wiley and IEEE Press, 2003, 1060 pages.

[2] L. Hanzo, M. Münster, B. J. Choi, and T. Keller, $O F D M$ and $M C-C D M A$. John Wiley and IEEE Press, 2003, 970 pages.

[3] S. L. Miller and B. J. Rainbolt, "MMSE Detection of Multicarrier CDMA," IEEE Journal on Selected Areas in Communications, vol. 18, pp. 2356-2362, Nov 2000.

0-7803-8255-2/04/\$20.00 @2004 IEEE.
[4] P. Zong, K. Wang, and Y. Bar-Ness, "Partial Sampling MMSE Interference Suppression in Asynchronous MultiCarirrer CDMA System," IEEE Journal on Selected Areas in Communications, vol. 19, pp. 1605-1613, August 2001.

[5] X. Gui and T. S. Ng, "Performance of Asynchronous Orthogonal Multicarrier CDMA Systen in Freqencey Selective Fading Channel," IEEE Transactions on Communications, vol. 47, pp. 1084-1091, July 1999.

[6] X. Wang and H. V. Poor, "Blind Multiuser, Detection: A Subspace Approach," IEEE Transactions on Information Theory, vol. 44, pp. 677-690, March 1998.

[7] X. Wang and H. V. Poor, "Blind Equalizaton and Multiuser Detection in Dispersive CDMA Channels," IEEE Transactions on Communications, vol. 6, pp. 91-103, Jan 1998.

[8] X. Wang and A. Host-Madsen, "Group-Blind Multiuser Detection for Uplink CDMA," IEEE Journal on Selected Areas in Communications, vol. 17, pp. 1971-1984, Nov 1999.

[9] A. Host-Madsen and X. Wang, "Performance of Blind and Group-Blind Multiuser Detection," IEEE Transactions on Information Theory, vol. 48, pp. 1849-1872, July 2002 .

[10] M. Wax and T. Kailath, "Detection of Signals by Information Theroretic Criteria," IEEE Transactions on Acoustics, Speech and Signal Processing, vol. 33, pp. $387-392$, April 1985.

[11] D. Chu, "Polyphase Codes with Good Periodic Correlation Properties," IEEE Transactions on Information Theory, vol. 18, pp. 531 - 532, July 1972.

[12] R. L. Peterson, R. E. Ziemer, and D. E. Borth, Introduction to Spread Spectrum Communications. Prentice Hall International Editions, 1995.

[13] S. Zhou, B. Muquet, and G. B. Giannakis, "Subspace-Based (Semi-) Blind Channel Estimation for Block Precoded Space-Time OFDM," IEEE Transactions on Signal Processing, vol. 50, pp. 12151228, May 2002. 